TITLE:

\title{
Mechanical properties and their influence to transport property of multifilamentary Bi2223 tape
}

$\operatorname{AUTHOR}(S):$

Osamura, K; Sugano, M; Matsumoto, K

\section{CITATION:}

Osamura, K ... [et al]. Mechanical properties and their influence to transport property of multifilamentary Bi2223 tape. IEEE TRANSACTIONS ON APPLIED SUPERCONDUCTIVITY 2003, 13(2): 3540-3543

ISSUE DATE:

2003-06

URL:

http://hdl.handle.net/2433/50190

\section{RIGHT:}

(c)2003 IEEE. Personal use of this material is permitted. However, permission to reprint/republish this material for advertising or promotional purposes or for creating new collective works for resale or redistribution to servers or lists, or to reuse any copyrighted component of this work in other works must be obtained from the IEEE. 


\title{
Mechanical Properties and Their Influence to Transport Property of Multifilamentary Bi2223 Tape
}

\author{
Kozo Osamura, Michinaka Sugano, and Kaname Matsumoto
}

\begin{abstract}
In order to investigate mechano-electromagnetic property, a structure model is proposed based on the rule of mixture. A comparison with experiments made clear that the present tapes show a three stage behavior. The first elastic region (stage I') is very narrow and followed by stage II', where the slope of stress - elongation curve decreased gradually. Then the macroscopic yield and the plastic deformation region (stage III) appeared. A discernible degradation of critical current was observed at stages I' and II'. This degradation is nearly reversible and vanishes when the external tensile load was reduced to zero. At stage III, the critical current decreased abruptly and largely due to generation of macroscopic cracks.
\end{abstract}

Index Terms-Bi2223, compressive stress, critical current, tensile test.

\section{INTRODUCTION}

$\mathbf{M}$ ULTIFILAMENTARY Bi2223 tapes are now scheduled to be widely applied as power devices like transmission cable, motor and transformer and so on. Further improvement of superconducting and mechanical properties is, however highly requisite for commercial applications. Degradations of their superconducting and mechanical properties under operation are known to cause serious problems, because critical current depends sensitively on bending and tensile stresses, electromagnetic force, and mechanical and thermal repeating forces. Therefore the knowledge of mechanical property and its influence to the transport property is crucial for the practical application of superconducting materials. Their microstructure dependence has been investigated by several authors [1], [2]. Recently we also reported that the tapes consisting of mono oxide-filament show a three stage behavior [3]. There is an intermediate stage between the elastic and plastic regions, where the degradation of critical current takes place. It is suggested to relate to the initiation of microcrack in the oxide layer. Further in the plastic region, the oxide layer took place the multiple fracture [4].

In the present study, tensile tests were carried out at room temperature and at $77 \mathrm{~K}$ for three commercial mutlifilamentary Bi2223 tapes, while the critical current measurements under tensile load has been carried out at $77 \mathrm{~K}$. The microstructure dependence of mechanical property and its influence to the transport property has been investigated to elucidate how the microstructure affects physical properties.

Manuscript received August 5, 2002.

The authors are with the Department of Materials Science and Engineering, Kyoto University, Sakyo-ku, Kyoto 606-8501, Japan (e-mail: osamura@ hightc.mtl.kyoto-u.ac.jp; matsu@ hightc.mtl.kyoto-u.ac.jp; msugano@kumax. mtl.kyoto-u.ac.jp,).

Digital Object Identifier 10.1109/TASC.2003.812391

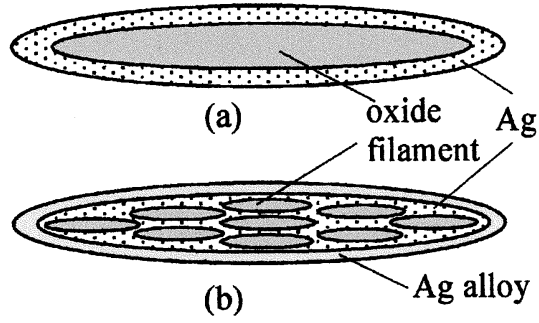

Fig. 1. Model of two type of metal sheath composite superconductors.

\section{THEORETICAL CONSIDERATION}

Firstly the construction of the PIT-processed tapes is schematically shown surveyed in Fig. 1(a) The preliminary monofilamentary tape consists of the oxide core embedded in the pure silver. The oxide core is so brittle and the pure silver is so soft that the yield stress is very low. Therefore many efforts have been paid to strengthen those original tapes. Fig. 1(b) shows a schematic view of cross section of the multifilamentary tape developed for industrial use. Both tapes might be treated as two and three component system in the viewpoint of mechanical property, respectively.

After heat treatment at a high temperature $\left(T_{A}\right)$, the tape is cooled down to room temperature $\left(T_{R T}\right)$. The tape shrinks with an average thermal expansion coefficient $\left(\alpha_{c}\right)$, and there the residual strain remains in each component. For the three component system of oxide(1) $-\mathrm{Ag}(2)-\mathrm{Ag}$ alloy(3), the residual strain $\left(A_{\mathrm{ri}}\right)$ and stress $\left(R_{\mathrm{ri}}\right)$ are given by the equations, respectively; for the oxide layer, the component is elastic,

$$
\begin{aligned}
& A_{r 1}=\left(\alpha_{c}-\alpha_{1}\right)\left(T_{R T}-T_{A}\right) \\
& R_{r 1}=A_{r 1} E_{1}
\end{aligned}
$$

for the Ag matrix, the component is plastically deformed,

$$
\begin{aligned}
& A_{r 2}=\left(\alpha_{c}-\alpha_{2}\right)\left(T_{R T}-T_{A}\right) \\
& R_{r 2}=\left(1-\omega_{2}\right) R_{y 2}+\omega_{2} A_{r 2} E_{2}
\end{aligned}
$$

for the Ag alloy matrix, the component is elastic,

$$
\begin{aligned}
& A_{r 3}=\left(\alpha_{c}-\alpha_{3}\right)\left(T_{R T}-T_{A}\right) \\
& R_{r 3}=A_{r 3} E_{3}
\end{aligned}
$$

where $E$ is the elastic constant, $\omega$ is the work hardening coefficient and $R_{y}$ is the yield stress. Without any external force, the total stress is balanced as follows;

$$
V_{1} R_{r 1}+V_{2} R_{r 2}+V_{3} R_{r 3}=0
$$




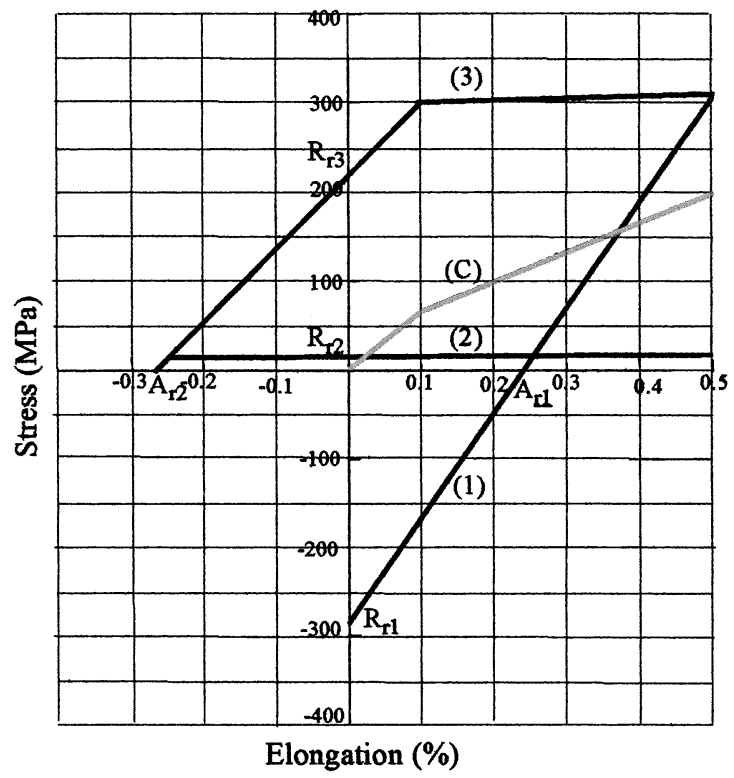

Fig. 2. Stress - elongation relationship appearing in three component system Here the symbols (1), (2), (3) and (c) indicates the oxide, silver, silver alloy component and the whole composite, respectively.

TABLE I

SPECIFICATION OF SAMPLES TESTED HERE

\begin{tabular}{l|c|c|c}
\hline Sample & Matrix & $\begin{array}{c}\text { Filament } \\
\text { No. }\end{array}$ & $\begin{array}{c}\text { Volume Fraction } \\
\text { V(Bi2223/V(Ag)/V(Ag Alloy) }\end{array}$ \\
\hline VAM1 & $\mathrm{Ag} / \mathrm{Ag}-\mathrm{Mg}$ & 57 & $0.27 / 0.40 / 0.33$ \\
\hline VAM2 & $\mathrm{Ag} / \mathrm{Ag}-\mathrm{Mg}-\mathrm{Ni} / \mathrm{ZrO}_{2}$ & 19 & $0.25 / 0.34 / 0.41$ \\
\hline VAM3 & $\mathrm{Ag} / \mathrm{Ag}-\mathrm{Mg}-\mathrm{Ni}$ & 37 & $0.29 / 0.35 / 0.36$ \\
\hline
\end{tabular}

where $V_{i}$ is the volume fraction of component $i$. The average thermal expansion coefficient is expressed as follows;

$$
\begin{aligned}
\alpha_{c}=\frac{\left(1-\omega_{2}\right) R_{y 2} V_{2}}{\left[\left(T_{A}-T_{R T}\right)\left(E_{1} V_{1}+\omega E_{2} V_{2}+E_{3} V_{3}\right)\right]} \\
+\frac{\left(\alpha_{1} E_{1} V_{1}+\omega_{2} \alpha_{2} E_{2} V_{2}+\alpha_{3} E_{3} V_{3}\right)}{\left(E_{1} V_{1}+\omega E_{2} V_{2}+E_{3} V_{3}\right)} .
\end{aligned}
$$

When we put the following physical parameters, the stress-extension relation is estimated as given in Fig. 2; $T_{A}=1103$, $T_{R T}=298[\mathrm{~K}], E_{1}=120, E_{2}=71, E_{3}=83[\mathrm{GPa}]$, $V_{1}=0.29, V_{2}=0.35, V_{3}=0.36, \alpha_{1}=15.4, \alpha_{2}=21.6, \alpha_{3}=$ $21.6 \times 10^{-6}\left[\mathrm{~K}^{-1}\right], R_{y 2}=11.2 \mathrm{MPa}, \omega_{2}=0.008, \omega_{3}=0.017$. Then, $A_{r 1}=0.236 \%, A_{r 2}=-0.262 \%, A_{r 2}=-0.262 \%$, $\alpha_{c}=18.34 \times 10^{-6}\left[\mathrm{~K}^{-1}\right], R_{r 1}=-283, R_{r 2}=12.84$, $R_{r 3}=217 \mathrm{MPa}$.

From Fig. 2, it should be noted that very large compressive stress $(-283 \mathrm{MPa})$ is present in the oxide component at the zero external force.

\section{EXPERIMENTAL PROCEDURE}

Three kinds of multifilamentary Bi2223 tapes have been investigated as listed in Table I. They are the commercially available tapes as supplied for the VAMAS program [5]. In general, their construction is similar to that shown in Fig. 1(b) and their volume fraction is listed in Table I.
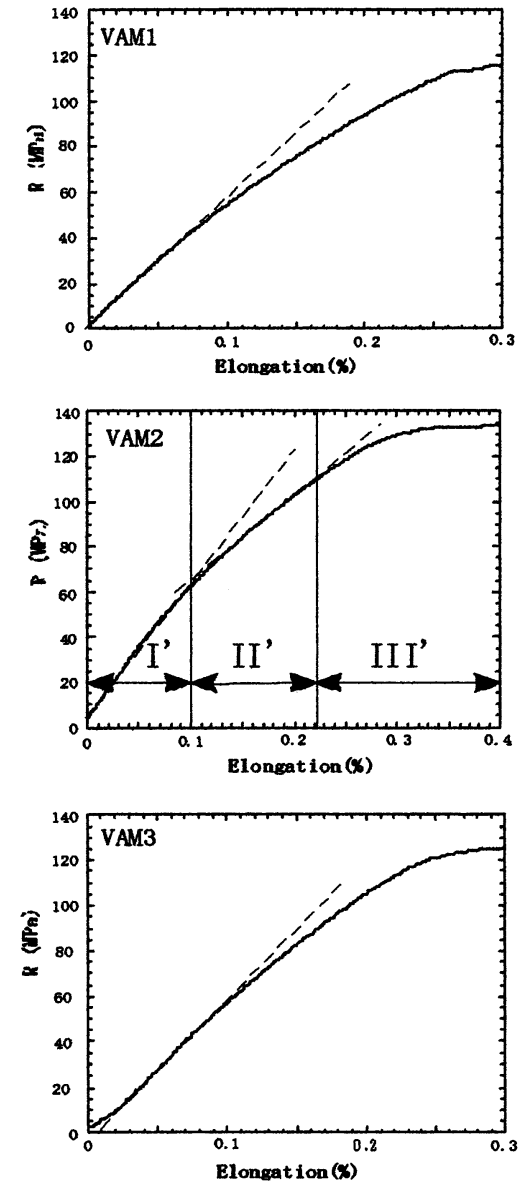

Fig. 3. Stress - percentage elongation curves for three typical Bi2223 multifilamentary tapes at room temperature.

The tensile test was carried out using an Instron-type machine (NMB TCM-500) at room temperature and in liquid nitrogen. The typical sample length was $70 \mathrm{~mm}$ and the distance between the chucks was $45 \mathrm{~mm}$. For the strain measurements, a couple of Nyilas type extensometers was used. The strain rate for the tensile test was $1.1 \times 10^{-4}$ per second. In order to measure simultaneously both mechanical property and $V-I$ characteristics, the current and voltage leads were attached on the tape sample.

The critical current measurement and the mechanical test were carried out at $77 \mathrm{~K}$ without external magnetic field. The critical current was determined with criterion of $1 \mu \mathrm{V} / \mathrm{cm}$.

\section{EXPERIMENTAL RESULTS AND DisCUSSION}

\section{A. Room Temperature Tensile Test}

Fig. 3 shows the initial part of stress-elongation curve for three tapes. When the maximum slope is drawn in each curve as shown by the dashed curve, it deviates approximately at a certain elongation between 0.08 and $0.1 \%$. After this, the slope decreases gradually and then saturates to the maximum stress level between 115 and $130 \mathrm{MPa}$ at $0.3 \%$ elongation. It is suggested that the elastic region is ranged up to about $0.1 \%$ elongation. This first part is called here stage I'. The detailed behavior has been already discussed elsewhere [5], [6]. Comparing with the theoretical estimation shown in Fig. 2, the change of slope takes place clearly at around $0.1 \%$ elongation. Therefore it is 


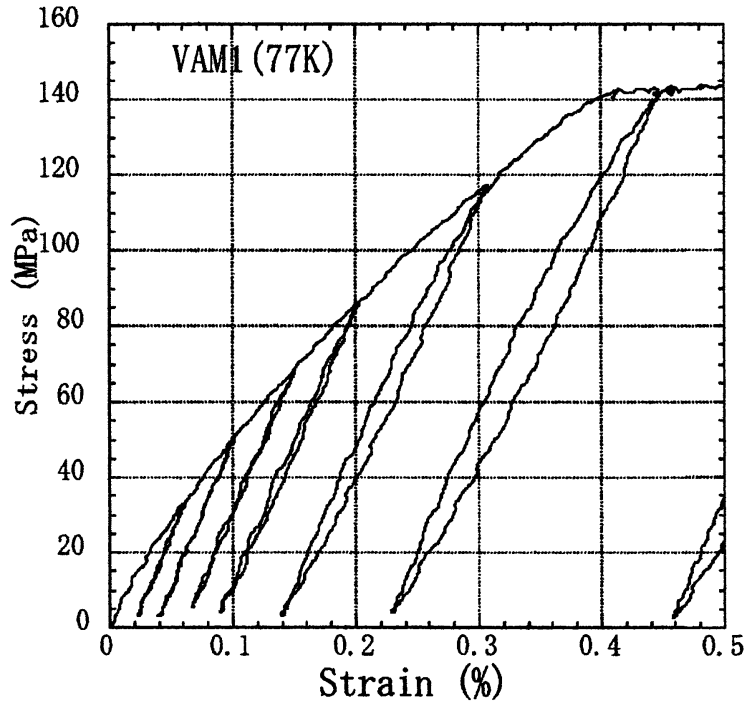

Fig. 4. Unloading - reloading curve for the sample VAM1.

now suggested that the Ag alloy component deforms plastically when the elongation exceeds $0.1 \%$ or the tensile stress increases beyond about $50-60 \mathrm{MPa}$.

The second slope can be drawn following stage I' as shown in Fig. 3 and again deviates from the observe stress elongation curve at about stress level of $110 \mathrm{MPa}$. This second range between 50 and $110 \mathrm{MPa}$ is called stage II'.

In order to ensure this elastic - plastic transition, the tensile load was reduced at a certain stress level $\left(R_{\text {unl }}\right)$ and again increased as shown in Fig. 4. When the stress level $R_{\text {unl }}$ is larger than $60 \mathrm{MPa}$, the hysteresis is observed in the unloading - reloading curve. This suggests that the Ag alloy component deforms elastically and plastically during the process just as predicted theoretically in the above section. Of course, the boundary between I' and II' stages is not rigid, but changes depending on geometrical factors like volume fraction of each constituent.

\section{B. Degradation of Critical Current}

In the next step, the $V-I$ characteristics was measured each time after the specimen was stressed. Then the critical current $I_{c}$ was evaluated as shown in Fig. 5.

The $I_{c}$ seems to start to decrease from the original point, but its decreasing rate is small in stage I' up to $50-60 \mathrm{MPa}$. Then the decreasing rate became larger gradually. Beyond $140 \mathrm{MPa}$, the critical current dropped down abruptly in stage III due to the macroscopic fracture in the oxide layer [4].

As shown in Fig. 2, the residual strain exerted in the oxide component is compressive and its value is $0.236 \%$, which is dependent on volume fraction of constituents. The degradation of $I_{c}$, on the other hand, starts from the zero external stress. The oxide component consists of stacking layers of Bi2223 phase as represented schematically in Fig. 6(a). The minor nonsuperconducting phases like $\mathrm{CuO}, \mathrm{Bi}$-free phase and so on, distribute in the layer. Their total volume fraction has been reported to be 7 to $10 \%$ [7]. In Fig. 6(a), a nonsuperconducting phase is represented in the center, of which the elastic constant $E_{1}^{*}$ is assumed to be larger than $E_{1}$ of Bi2223 phase.

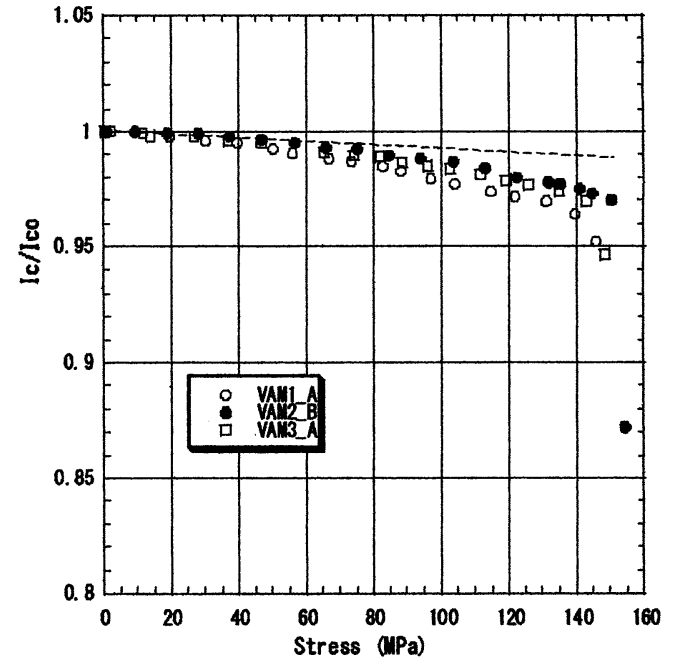

Fig. 5. Change of relative critical current as a function of tensile stress for three tapes.

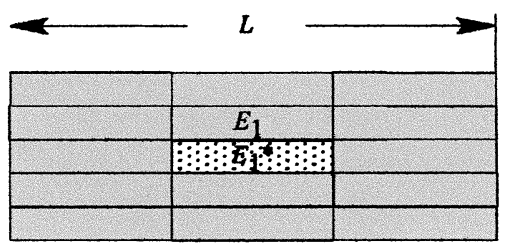

(a)

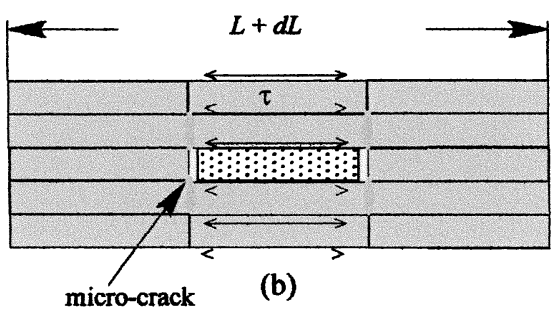

Fig. 6. Schematic illustration how the microcracks generate under the compressive stress.

When the tensile load is applied, the considering area with length $L$ expands by an increment $\Delta L$. During this process, the shear stress is transmitted through the grain boundary from grain to grain Depending on the strength of interfacial bonding, the shear deformation behaves elastically or plastically [8]. The nonsuperconducting phase deforms by shear stress through the interface and then it expands. Its increment is proportional to the elastic constant $E_{1}^{*}$. In the present situation, the increment is smaller comparing with the surroundings and consequently a tensile stress generates locally at both end of its phase as shown in Fig. 6(b). Due to this local stress concentration, micro-cracks might generate and then the number of grain boundary weak link path decreases.

Fig. 7 shows the change of critical current as a function of elongation together with the master curve of stress - elongation relationship. When the tensile load was applied up to $R_{A}$ and unloaded to zero level, the change of $I_{c}$ was almost reversible. Here $R_{A}$ is about $100 \mathrm{MPa}$, which is lying still in Stage II'. According to the proposed model shown in Fig. 6, it should be 


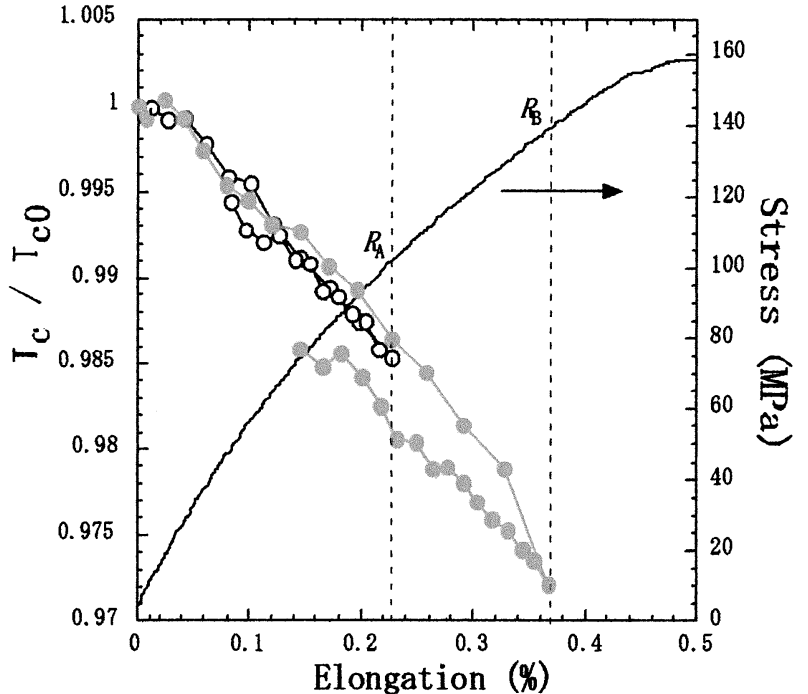

Fig. 7. Change of critical current during loading and unloading processes for tape VAM2.

noted that microcracks generating due to the relaxation of compressive stress vanish nearly when the external stress is reduced to zero.

When the tensile load was applied to $R_{B}$, on the other hand, the change of $I_{c}$ was irreversible. It is clear that macroscopic cracks generated in the oxide component just after applying tensile stress close to $140 \mathrm{MPa}$.

\section{CONCLUSION}

Multifilamentary Bi2223 tapes consist of three components of brittle oxide layer surrounded by pure Ag sheath embedded in the $\mathrm{Ag}$ alloy matrix. In order to investigate quantitatively tensile property and its influence to transport property, a structure model is proposed based on the rule of mixture. During cooling from reaction temperature, each component contracts, but its quantity is constrained from each other. At $77 \mathrm{~K}$, a compressive stress is exerted in the oxide layer and a tensile stress remains in the Ag alloy component while the pure Ag component has plastically deformed. When applying tensile load to this model system, a stress-elongation behavior can be easily drawn and compared with the experiments. This comparison made clear that the present tapes show a three stage behavior. The first elastic region (stage I') is very narrow and followed by stage II', where the slope of stress - elongation curve decreased gradually. Then the macroscopic yield and the plastic deformation region (stage III) appeared. The stress dependency of $V-I$ characteristics can be well explained in terms of three stage behavior. A discernible degradation of critical current was observed at stages I' and II'. Its degree was at most less than $3 \%$. This degradation is nearly reversible and vanishes when the external tensile load was reduced to zero. At stage III, the critical current decreased abruptly and largely due to generation of macroscopic cracks.

\section{ACKNOWLEDGMENT}

The authors express their hearty thanks to Dr H. Wada and Dr K. Itoh (NRIM, Japan) for their kind help on the VAMAS program.

\section{REFERENCES}

[1] S. Ochiai, K. Hayashi, and K. Osamura, "Improvement of strain endurance of critical current SC tapes by reducing volume fraction of oxide core," Cryogenics, vol. 33, pp. 976-979, 1993.

[2] M. Sugano, K. Osamura, and S. Ochiai, "Influence of dynamic and static stresses to mechanical and transport properties of $\mathrm{Ag} / \mathrm{Bi} 2223$ composite superconductors," IEEE Trans. Appl. Supercond., vol. 11, pp. 3022-3025, 2001.

[3] K. Osamura and S. Sugano, "Mechanical properties of PIT processed mono- and multi- filamentary Bi2223 tapes," Physica C, vol. 357-360, pp. 1128-1133, 2001.

[4] K. Osamura, M. Sugano, T. Wada, and S. Ochiai, "Mechanical properties of Ag/Bi2223 composite superconductors," Adv. Cryo. Eng., vol. 46, pp. 639-645, 2000.

[5] K. Osamura et al., "Tensile property of commercial Bi2223 tapes: Report on the international round-robin test," Supercond. Sci. Technol., vol. 15, pp. 1-6, 2002.

[6] K. Osamura and M. Sugano, "Three stage behavior in stress-elongation curve of multifilamentary Bi2223 tapes," Adv. Cryo. Eng., vol. 48, pp. 477-484, 2002.

[7] K. Osamura, S. Nonaka, and M. Matsui, "Non-superconducting phases and their influence on critical current density in Ag/Bi2223 tapes," Physica C, vol. 257, pp. 79-85, 1996.

[8] S. Ochiai, K. Hayashi, and K. Osamura, "Estimation of interfacial shear strength between superconducting oxides and silver sheath from multiple fracture phenomenon of the oxide," Metall.and Mater. Trans. A, vol. 25 , pp. 349-356, 1994. 